\title{
Electric-field control of the band gap and Fermi energy in graphene multilayers by top and back gates
}

\author{
A. A. Avetisyan, ${ }^{1,2, *}$ B. Partoens, ${ }^{1, \dagger}$ and F. M. Peeters ${ }^{1,3, *}$ \\ ${ }^{1}$ Departement Fysica, Universiteit Antwerpen, Groenenborgerlaan 171, B-2020 Antwerpen, Belgium \\ ${ }^{2}$ Department of Physics, Yerevan State University, 1 A. Manoogian, 0025 Yerevan, Armenia \\ ${ }^{3}$ Departamento de Física, Universidade Federal do Ceará, Caixa Postal 6030, Campus do Pici, 60455-900 Fortaleza, CE, Brazil
}

(Received 29 June 2009; revised manuscript received 11 September 2009; published 2 November 2009)

\begin{abstract}
It is known that a perpendicular electric field applied to multilayers of graphene modifies the electronic structure near the $K$ point and may induce an energy gap in the electronic spectrum which is tunable by the gate voltage. Here we consider a system of graphene multilayers in the presence of a positively charged top and a negatively charged back gate to control independently the density of electrons on the graphene layers and the Fermi energy of the system. The band structure of three- and four-layer graphene systems in the presence of the top and back gates is obtained using a tight-binding approach. A self-consistent Hartree approximation is used to calculate the induced charges on the different graphene layers. We predict that for opposite and equal charges on the top and bottom layers an energy gap is opened at the Fermi level. For an even number of layers this gap is larger than in the case of an odd number of graphene layers. We find that the circular asymmetry of the spectrum, which is a consequence of the trigonal warping, changes the size of the induced electronic gap, even when the total density of the induced electrons on the graphene layers is low.
\end{abstract}

DOI: 10.1103/PhysRevB.80.195401

PACS number(s): 81.05.Uw, 73.63.Bd, 73.43.Cd

\section{INTRODUCTION}

Since the fabrication of single and multilayers of graphene, ${ }^{1}$ the investigation of its exotic electronic, optical, and transport properties became an intriguing area in contemporary physics. Ultrathin graphite films are very promising for, e.g., nanoelectronics ${ }^{2}$ and as transparent conducting layers ${ }^{3}$ which is important for, e.g., displays and solar cells.

In such systems magnetotransport was measured and the integer quantum Hall effect was observed., ${ }^{1,4}$ It was concluded that a single graphene layer has a $k$-linear Dirac-like spectrum $^{1,4,5}$ while quasi-two dimensional highly oriented pyrolytic graphite has both parabolic and Dirac-like dispersion with massless electrons. ${ }^{6}$

Theoretical and experimental investigations have shown that an applied electric field, directed perpendicularly to bilayer graphene, can open an electronic gap between the valence and conduction bands. ${ }^{7-9}$ Recently it was shown that by applying a perpendicular electric field a tunable energy gap can also be opened in three- and four-layer graphene systems ${ }^{10}$ whose size depends on the number of layers. The circular asymmetry of the band structure, that is a consequence of trigonal warping, leads to a nonmonotonic behavior of the induced gap in multilayers of graphene. ${ }^{10}$ In Ref. 10 it was found that the Fermi energy is located outside the induced energy gap.

In Ref. 11 the electronic band structure of $A B A$-stacked trilayer graphene was studied self-consistently, in the field of back and top gates, to estimate the conductivity of trilayer graphene. The behavior of the induced gap was not systematically studied in Ref. 11 and the results were limited to the case in which there is a net electron charge on graphene. We generalize these results to the case of hole charges and found that the obtained results do not exhibit electron-hole symmetry.

In the present paper we generalize our previous results ${ }^{10}$ and study the band structure of three and four layers of graphene in the presence of top and back gates taking into account the effect of trigonal warping, which we found leads to a stronger modification of the band structure as compared to the case when only a single gate is applied. We predict a nonmonotonic behavior of the true energy gap in trilayer graphene as a function of the top gate density, when charges on the top and the back gates are opposite but equal in magnitude. This aspect was not discussed in Ref. 11. We found also an indirect gap with a similar nonmonotonic character, which at low and intermediate charge densities on the gates is smaller than the direct true gap while for larger densities both gaps coincide. For four-layer graphene we did not observe such a nonmonotonic behavior, neither an indirect gap. Our analysis is based on a tight-binding approach to calculate the band structure of multilayers of graphene and a selfconsistent Hartree approximation is used to find the induced charges on the different graphene layers when an external gate voltage is applied.

In our previous work ${ }^{10}$ with a single gate, we found that the true energy gap tends to decrease with increasing number of graphene layers. Here we find that when the charges on the top and back gates are opposite but equal in magnitude, the four graphene layer system may open up a larger gap than the three-layer system. This can be explained as being a consequence of the fact that Dirac fermions are present in $A B$-stacked graphene multilayers in case of an odd number of layers while for an even number of stacked graphene layers only charge carriers with a parabolic energy dispersion are present. ${ }^{12}$

Such three-layer graphene samples can be realized experimentally as was recently demonstrated in Ref. 13 where transport measurements on a tunable three-layer graphene single-electron transistor were reported and its functionality was proven through Coulomb blockade oscillations. It is expected that in the near future similar experiments will be performed on four layers of graphene. 
This paper is organized as follows. The details of our tight-binding approach with the description of the selfconsistent calculation are given in Sec. II for the three and bilayer graphene systems in the presence of two gates, and the corresponding results are discussed in Sec. II A for the bilayer and in Sec. II B for the three-layer case. In Sec. II C we discuss results for three-layer graphene when only one, i.e., the back or top gate is applied. In Sec. III we investigate a four-layer graphene system in the presence of top and back gates, and Sec. IV summarizes our conclusions.

\section{THREE AND BILAYER GRAPHENE SYSTEMS IN AN EXTERNAL ELECTRIC FIELD}

We consider a system consisting of three layers of graphene, which is modeled as three coupled hexagonal lattices with inequivalent sites $A_{i}$ and $B_{i}$ in the $i$ th layer, with $A_{i}$ and $A_{i+1}$ atoms in adjacent layers on top of each other. A top gate with a density of negative charges $n_{t}>0$ (the electron excess density is positive) on it and a back gate with a density of positive charges $n_{b}<0$ are applied to control the density of electrons on the different graphene layers and the Fermi energy of the system; the system is schematically shown in Fig. 1. As a result in the graphene system a total excess density $n=n_{1}+n_{2}+n_{3}$ is induced $\left(n=n_{t}+n_{b}\right)$, where $n_{1}$ is the excess density on the closest layer to the top gate and $n_{2}\left(n_{3}\right)$ is the excess density on the second (third) layer from the top gate. In our model the top or back gate produces a uniform electric field $E_{t, b}=n_{t, b} e / 2 \varepsilon_{0} \kappa$, where $\varepsilon_{0}$ is permittivity of the vacuum and $\kappa$ is the dielectric constant. For our numerical calculations we use the value $\kappa=2.3$, which corresponds to graphene layers on $\mathrm{SiO}_{2}$ as well as $\kappa=1$, which describes freely suspended graphene in vacuum. There is a simple relation between the charge density on the gates and the voltage between the gate and the closest graphene layer: $V_{t, b}=e n_{t, b} d / 2 \varepsilon_{0} \kappa$, where $d$ is the distance from a gate to a closest graphene layer ( $d$ is equal to the oxide thickness, which is usually about $300 \mathrm{~nm}$ ). The charges in the layers of graphene, in its turn, produce a uniform electric field $E_{i}$ $=n_{i} e / 2 \varepsilon_{0} \kappa$, where $i=1,2,3$ is the layer number. The layer asymmetries between first and second layers, as well as between second and third layers are determined by a corresponding change in the potential energy $\Delta_{1,2}$ and $\Delta_{2,3}$

$$
\begin{gathered}
\Delta_{1,2}(n)=\alpha\left(n_{2}+n_{3}-\left|n_{b}\right|\right), \\
\Delta_{2,3}(n)=\alpha\left(n_{3}-\left|n_{b}\right|\right) .
\end{gathered}
$$

where $\alpha=e^{2} c_{0} / \varepsilon_{0} \kappa$, with $c_{0}=3.35 \AA$ the interlayer distance. In order to obtain the band structure in the presence of the electric field we should add $-\Delta_{1,2}(n)$ and $\Delta_{2,3}(n)$ to the first and third layer on-site elements of the three-layer system Hamiltonian in the absence of gates. ${ }^{14}$ The tight-binding Hamiltonian for the three $A B A$-stacked graphene layers in the presence of the top and back gates becomes

$$
H=\left(\begin{array}{cccccc}
-\Delta_{1,2}(n)+\Delta+\gamma_{5} & \gamma_{0} f & \gamma_{1} & -\gamma_{4} f^{*} & \gamma_{5} / 2 & 0 \\
\gamma_{0} f^{*} & -\Delta_{1,2}(n)+\gamma_{2} & -\gamma_{4} f^{*} & \gamma_{3} f & 0 & \gamma_{2} / 2 \\
\gamma_{1} & -\gamma_{4} f & \Delta+\gamma_{5} & \gamma_{0} f^{*} & \gamma_{1} & -\gamma_{4} f \\
-\gamma_{4} f & \gamma_{3} f^{*} & \gamma_{0} f & \gamma_{2} & -\gamma_{4} f & \gamma_{3} f^{*} \\
\gamma_{5} / 2 & 0 & \gamma_{1} & -\gamma_{4} f^{*} & \Delta_{2,3}(n)+\Delta+\gamma_{5} & \gamma_{0} f \\
0 & \gamma_{2} / 2 & -\gamma_{4} f^{*} & \gamma_{3} f & \gamma_{0} f^{*} & \Delta_{2,3}(n)+\gamma_{2}
\end{array}\right),
$$

where the rows and columns are ordered according to atom $A$ from layer 1, atom $B$ from layer 1, atom $A$ from layer 2, atom $B$ from layer 2, etc. In Eq. (3) $\gamma_{0}, \gamma_{1}, \gamma_{2}, \gamma_{3}, \gamma_{4}, \gamma_{5}$ and $\Delta$ are the Slonczewski-Weiss-McClure parameters and the function $f$ stands for

$$
f\left(k_{x}, k_{y}\right)=e^{i k_{x} a_{0} / \sqrt{3}}+2 e^{-i k_{x} a_{0} / 2 \sqrt{3}} \cos k_{y} a_{0} / 2,
$$

with $a_{0}=2.46 \AA$ the length of the in-plane lattice vector. The six parameters $\gamma_{0}, \gamma_{1}, \gamma_{2}, \gamma_{3}, \gamma_{4}$, and $\gamma_{5}$ express the couplings between the different atoms and are given in Ref. 10.

The tight-binding Hamiltonian operates in the space of coefficients of the tight-binding functions $c(\vec{k})$ $=\left(c_{A_{1}}, c_{B_{1}}, c_{A_{2}}, c_{B_{2}}, c_{A_{3}}, c_{B_{3}}\right)$, where $c_{A_{i}}=c_{A_{i}}(\vec{k})$ and $c_{B_{i}}$ $=c_{B_{i}}(\vec{k})$ are the $i$ th layer coefficients for $A$ and $B$ type of atoms, respectively. The total eigenfunction of the system is then given by

$$
\Psi_{\vec{k}}(\vec{r})=\sum_{i=1}^{N_{l}} c_{A_{i}} \psi_{\vec{k}}^{A_{i}}(\vec{r})+\sum_{i=1}^{N_{l}} c_{B_{i}} \psi_{\vec{k}}^{B_{\vec{k}}}(\vec{r}),
$$

with $N_{l}$ the number of layers. The six coefficients in Eq. (5) of the three-layer system, for fixed values of the layer asymmetries defined by Eqs. (1) and (2), can be obtained by diagonalizing Eq. (3). The electronic densities on the individual layers are given by

$$
n_{i}=\frac{2}{\pi} \int d k_{x} d k_{y}\left(\left|c_{A_{i}}\right|^{2}+\left|c_{B_{i}}\right|^{2}\right) .
$$

Since the coefficients $c_{A_{i}}$ and $c_{B_{i}}$ depend also on the specific band, we can distinguish the electronic densities on each band. The following cases are possible: when the magnitudes of the top and back gates are equal to each other (but have opposite charges on them) then the Fermi energy is located 

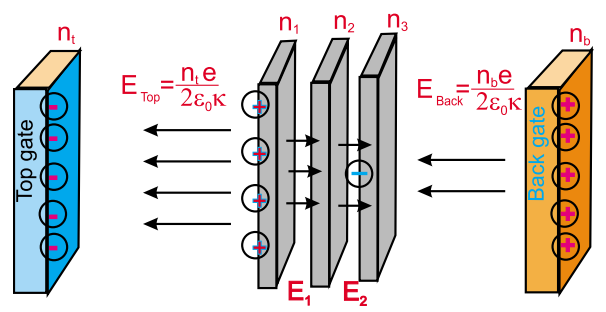

FIG. 1. (Color online) Schematic of the three-layer graphene system, near a negatively charged top gate with a charge density $n_{t}>0$ and a positively charged back gate with a density $n_{b}<0$, which induce a total excess density $n=n_{1}+n_{2}+n_{3}$ on the graphene layers, with $n_{1}$ the excess density of electrons on the closest layer to the top gate and $n_{2}\left(n_{3}\right)$ is the excess density on the second (third) layer; $E_{i}(i=1,2)$ is the uniform electric field between the layers with $E_{1}=\left(n_{2}+n_{3}-\left|n_{b}\right|\right) e / \varepsilon_{0} \kappa$ and $E_{2}=\left(n_{3}-\left|n_{b}\right|\right) e / \varepsilon_{0} \kappa$.

in the opened band gap; in this case to find the electron densities in the valence bands one should integrate Eq. (6) from zero until some optimal $k_{\max }$ (which is chosen such that the energy gap is convergent). In the case when the density of the positive charge in the back gate is larger than the density of the negative charge on the top gate (or in the absence of the top gate) the Fermi energy will be located in the conduction band. After integrating Eq. (6) till the Fermi vector $k_{F}$ we find the charge densities in the partially occupied bands, namely, in the first and second conduction bands. To take into account the density redistribution in the valence bands one should integrate Eq. (6) from zero until some large $k_{\max }$. Using Eqs. (1)-(3) and (6) we evaluate the energy gap $\Delta_{0}$ at the $K$ point and the true gap, $\widetilde{\Delta}$, self-consistently for a fixed total density $n_{t}+n_{b}=n_{1}+n_{2}+n_{3}$ (see Refs. 7 and 10).

For comparison purposes we include here also the results for bilayer graphene where top and bottom are at a different gate potential. The tight-binding Hamiltonian for a bilayer graphene, with layer asymmetry between first and second layers $\Delta_{1,2}(n)=\alpha\left(n_{2}-\left|n_{b}\right|\right)$, in the presence of the top and back gates is reduced to

$$
H=\left(\begin{array}{cccc}
-\Delta_{1,2}(n) / 2 & \gamma_{0} f & \gamma_{1} & -\gamma_{4} f^{*} \\
\gamma_{0} f^{*} & -\Delta_{1,2}(n) / 2 & -\gamma_{4} f^{*} & \gamma_{3} f \\
\gamma_{1} & -\gamma_{4} f & \Delta_{1,2}(n) / 2 & \gamma_{0} f^{*} \\
-\gamma_{4} f & \gamma_{3} f^{*} & \gamma_{0} f & \Delta_{1,2}(n) / 2
\end{array}\right) .
$$

The electronic densities on the individual layers for bilayer graphene are given by Eq. (6) and the gaps can be calculated self-consistently similarly as indicated above for the threelayer case.

In the following we will consider two cases. First, we neglect all interactions except between the nearest-neighbor atoms in the same layer and between $A$-type atoms of adjacent layers (which are on top of each other), i.e., we put $\gamma_{2}$ $=\gamma_{3}=\gamma_{4}=\gamma_{5}=0$. In our calculations we used the parameter $\gamma_{0}=3.12 \mathrm{eV}$ which within each plane leads to an in-plane velocity $v=\sqrt{3} \gamma_{0} a / 2 \hbar \simeq 10^{6} \mathrm{~m} / \mathrm{s}$ and for the interlayer coupling strength, i.e., between $A_{i}$ and $A_{i+1}$ atoms we take $\gamma_{1}$ $=0.377 \mathrm{eV}$ (see Ref. 14) and for the interlayer distance $c_{0}$
$=3.35 \AA$ A. Second, the full interaction case is studied. When all the interactions between the different atoms, which are expressed by the SWMcC parameters $\left(\gamma_{2}=-0.0206, \gamma_{3}\right.$ $\left.=0.29, \gamma_{4}=0.12, \gamma_{5}=0.025\right)$ are taken into account the energy surface is no longer circular. The corresponding results for these two cases for different number of graphene layers are discussed in next sections.

\section{A. Bilayer graphene in the presence of top and back gates}

The dependence of the first conduction-band minimum, the highest valence-band maximum, and the Fermi energy on the charge density of the back gate $n_{b}$ is shown in Fig. 2(b) for bilayer graphene, when only $\gamma_{0}, \gamma_{1}$ are taken into account (with $\kappa=1$ ) for fixed charge density of the top gate $n_{t}$ $=10^{12} \mathrm{~cm}^{-2}$. For equal magnitude of top and back gates the Fermi energy is located in the forbidden gap. Figure 2(a) shows the band structure for bilayer graphene when charges on the top and back gates are opposite but equal in magnitude with $\left|n_{b}\right|=n_{t}=10^{13} \mathrm{~cm}^{-2}$. Notice that the true gap $\tilde{\Delta}$ occurs away from the $K$ point where the gap is $\Delta_{0}$ $=294 \mathrm{meV}>\widetilde{\Delta}=232 \mathrm{meV}$. In Figs. 2(c) and 2(d) we show the dependence of the gap $\Delta_{0}$ at the $K$ point (dot-dashed curve), the true direct gap $\tilde{\Delta}$ (solid curve) for bilayer graphene $(\kappa=1)$ when including the full interaction with the SWMcC parameters, as a function of the top gate density $n_{t}$ with equal but opposite in sign back gate density $n_{b}=-n_{t}$. For comparison in Fig. 2(c) we show also the corresponding results, $\Delta_{0}^{\prime}$ (dashed curve) and $\widetilde{\Delta^{\prime}}$ (dotted curve) when only $\gamma_{0}, \gamma_{1} \neq 0$. In a recent experiment, ${ }^{15}$ slightly different values for the $\gamma$ parameters were obtained, i.e., $\gamma_{0}=2.9, \gamma_{1}$ $=3.0, \gamma_{3}=1.0, \gamma_{4}=1.2$. The values of the parameters of the SWMcC model for bilayer graphene in Ref. 15 were obtained from an analysis of the dispersive behavior of the Raman features, where the electronic structure of bilayer graphene was investigated from a resonant Raman study of the $G^{\prime}$ band using different laser excitation energies. For comparison reasons we give the obtained energy gap with these $\gamma$ parameters by the dashed (at the $K$ point) and dotted (true direct-gap) curves in Fig. 2(d).

We have found that even for low densities $\left(\left|n_{b}\right|=n_{t}\right.$ $\approx 10^{12} \mathrm{~cm}^{-2}$ ) it is important to take into account all the interactions between the atoms, and the true gap for this case is $\widetilde{\Delta}=20.3 \mathrm{meV}$ and the relative difference with the case when only $\gamma_{0}, \gamma_{1} \neq 0$ is about $10 \%$ [see Fig. 2(c)]. The relative difference between the results obtained in the present paper with the SWMcC parameters and the gaps found using the new $\gamma$ parameters proposed in Ref. 15 is about $10 \%$ for intermediate densities [see Fig. 2(d)]. Notice that the true gaps for both choices practically coincide at large densities. We were able to fit the different energy gaps for bilayer graphene using the different tight-binding parameters by a polynomial $\Delta(\mathrm{meV})=A \times n_{t}+B \times n_{t}^{2}$ (where $n_{t}$ in units of $10^{12} \mathrm{~cm}^{-2}$ ). The values of the parameters $A$ and $B$ for these different cases are given in Table I. For the case when only the back gate was applied the energy gap differs substantially at high densities and for $n=10^{13} \mathrm{~cm}^{-2}$ the relative difference was about $15 \% .{ }^{10}$ 

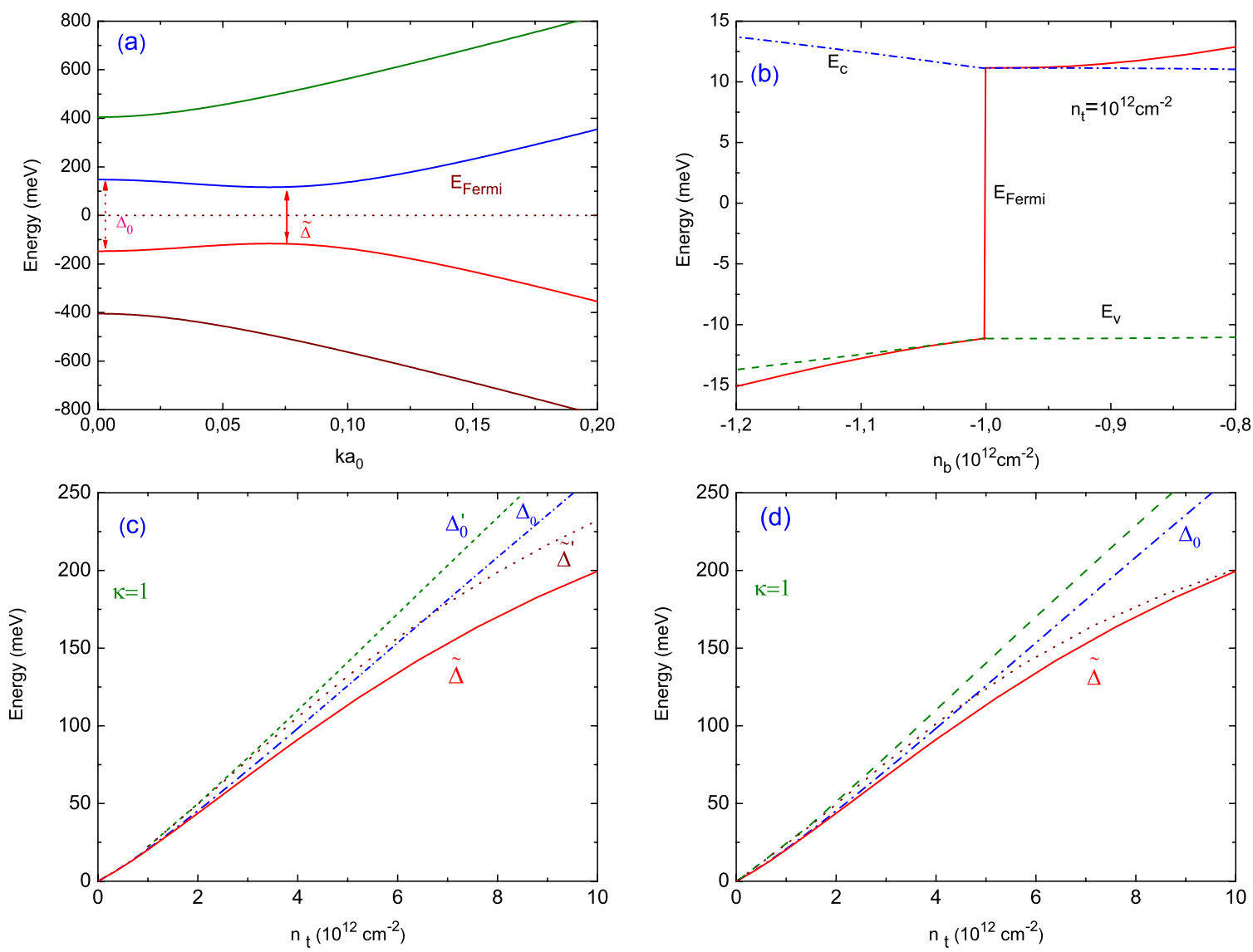

FIG. 2. (Color online) Results for bilayer graphene $(\kappa=1)$ around the $K$ point when only $\gamma_{0}, \gamma_{1} \neq 0$ : (a) Band structure for $-n_{b}=n_{t}$ $=10^{13} \mathrm{~cm}^{-2}$. Horizontal dotted line is the Fermi level. The true gap $\widetilde{\Delta}$ and the energy gap at the $K$-point $\Delta_{0}$ are indicated. (b) The dependence of the lowest conduction-band minimum (dot-dashed curve), highest valence-band maximum (dashed curve), and the Fermi energy (solid curve) on the density of the back gate $n_{b}$ with fixed density on the top gate $n_{t}=10^{12} \mathrm{~cm}^{-2}$. (c) The dependence of the gap $\Delta_{0}^{\prime}$ at the $K$ point, the true direct gap $\widetilde{\Delta^{\prime}}$ as a function of the top gate density $n_{t}$ when $n_{b}=-n_{t}$. $\Delta_{0}$ (dot-dashed curve) and $\widetilde{\Delta}$ (solid curve) are the results when including the full interaction with the SWMcC parameters. (d) The same as (c) but now with the $\gamma$ parameters from Ref. 15.

\section{B. Three-layer graphene in the presence of top and back gates}

When two gates, i.e., a top gate and a back gate are applied to three-layer graphene, it will allow us to control independently the opening of the gap and to change the Fermi level. However, for the three-layer system when only $\gamma_{0}, \gamma_{1}$ $\neq 0$ we found zero true direct and indirect gaps for any strength of the top gate (for equal magnitude of charge density on the top and back gates).

In contrast, for the full interaction case the symmetry of the energy spectrum is no longer circular but becomes trian- gular and it is possible to have an indirect true gap $\Delta_{k k^{\prime}}$. The behavior of the true indirect gap $\Delta_{k k^{\prime}}$ (solid curve) as well as the true direct gap $\widetilde{\Delta}$ (dot-dashed curve) and the $K$ gap $\Delta_{0}$ (dotted curve) are shown in Fig. 3(a) for $\kappa=1$ as a function of the strength of the top gate, provided that charges on the top and back gates are opposite but equal in magnitude. In this case we found for all values of the density that the Fermi energy is located in the energy gap and the total average electron density on the layers is zero. In fact we have an electron-hole bilayer system where the electrons (holes) are

TABLE I. The fitting parameters $A$ and $B$ for the density dependence of the energy gap $\Delta(\mathrm{meV})=A$ $\times n_{t}+B \times n_{t}^{2}$, where $n_{t}$ is in units of $10^{12} \mathrm{~cm}^{-2}$ with equal (but opposite in sign) density on top and bottom gates. Results are given for the true gap $\widetilde{\Delta}$ and the gap at $K$-point $\Delta_{0}$ for bilayer graphene, when all the SWMcC parameters are included, when only $\gamma_{0}, \gamma_{1}$ are taken into account (results with accent), when $\gamma$ parameters are taken from Ref. 15 (double accent results).

\begin{tabular}{lcccccc}
\hline \hline & $\begin{array}{c}\widetilde{\Delta} \\
(\mathrm{meV})\end{array}$ & $\begin{array}{c}\Delta_{0} \\
(\mathrm{meV})\end{array}$ & $\begin{array}{c}\widetilde{\Delta^{\prime}} \\
(\mathrm{meV})\end{array}$ & $\begin{array}{c}\Delta_{0}^{\prime} \\
(\mathrm{meV})\end{array}$ & $\begin{array}{c}\widetilde{\Delta^{\prime \prime}} \\
(\mathrm{meV}) \text { Ref. 15 }\end{array}$ & $\begin{array}{c}\Delta_{0}^{\prime \prime} \\
(\mathrm{meV}) \text { Ref. 15 }\end{array}$ \\
\hline$A\left(\mathrm{meV} \mathrm{cm}^{2}\right)$ & 0.02461 & 0.02342 & 0.02851 & 0.02618 & 0.02885 & 0.02689 \\
$B\left(\mathrm{meV} \mathrm{cm}^{4}\right)$ & -0.00044 & 0.00031 & -0.00049 & 0.00036 & -0.00086 & 0.00019 \\
\hline \hline
\end{tabular}



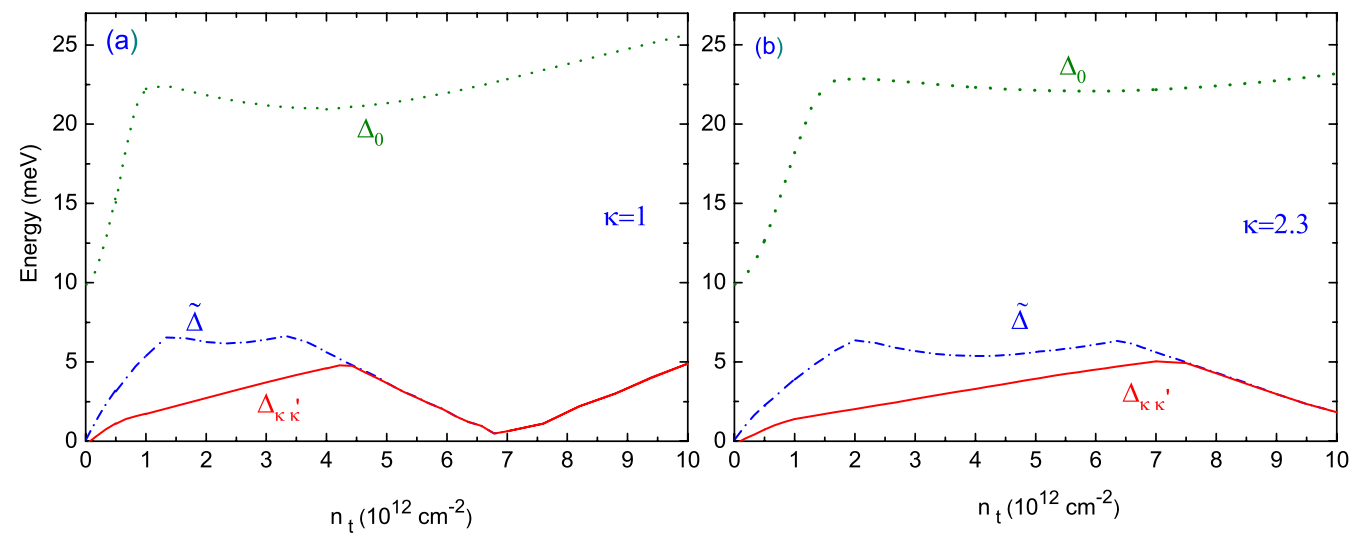

FIG. 3. (Color online) The dependence of the $K$-point gap $\Delta_{0}$ (dotted curve), the true direct gap $\widetilde{\Delta}$ (dot-dashed curve), and the true indirect gap (solid curve) $\Delta_{k k^{\prime}}$ as a function of the top gate density $n_{t}=-n_{b}$ for three-layer graphene where we included the full interaction. Results are shown for two cases: (a) $\kappa=1$ and (b) $\kappa=2.3$.

situated in the top (bottom) graphene layer and the middle layer has zero electron density. From Fig. 3(a) we notice that for low densities the indirect gap is smaller than the true direct gap and for $n_{t} \geq 4.5 \times 10^{12} \mathrm{~cm}^{-2}$ they coincide. The true direct gap becomes zero when $n_{t} \approx 2 \times 10^{13} \mathrm{~cm}^{-2}$ and it increases with the further increase in $n_{t}$. The equivalent results for the case of $\kappa=2.3$ are shown in Fig. 3(b). Notice that the results are qualitatively very similar but the point where the direct and indirect gaps coincide is shifted to $n_{t}$ $=7.5 \times 10^{12} \mathrm{~cm}^{-2}$. For $n_{t}<10^{13} \mathrm{~cm}^{-2}$ the maximum size of the energy gap is about $5 \mathrm{meV}$.

In order to gain a better understanding of the density dependence of the energy gap we show in Fig. 4 threedimensional (3D) plots and corresponding contourplots of the lowest conduction and the highest valence band for threelayer graphene near the $K$ point ( $K$ point is chosen as the origin and $\kappa=2.3$ ) for different values of $n_{t}$, providing $n_{b}=$ $-n_{t}$. For intermediate density $n_{t}=\left|n_{b}\right|=5 \times 10^{12} \mathrm{~cm}^{-2}$ the highest valence band exhibits several local maxima with the highest one situated at $k_{y}=0, k_{x}=-0.025$ and smaller local maxima with $k_{y} \neq 0, k_{x}=-0.025$ [see Fig. 4(a)]. The conduction band has corresponding minima in the same plane with $k_{x}=-0.025$ leading to an indirect gap, as shown in Fig. 5(a). Notice that the true direct as well as the indirect gap shown in the plane with $k_{x}=-0.025$ [Fig. 5(a)] equal the corresponding gaps found for $n_{t}=\left|n_{b}\right|=5 \times 10^{12} \mathrm{~cm}^{-2}$ in Fig. 3(b). At high densities the maxima and minima in the corresponding bands are comparable [see Fig. 4(b)] and the indirect gap coincides with the direct one, as shown in Fig. 5(b) for the plane with $k_{x}=-0.05$. Notice that for this density the minimal gap is located in the plane $k_{x}=-0.05$. The Fermi energy for all the densities is located in the energy gap and is indicated by the dotted curves in Fig. 5 .

We also show in Fig. 6 the energy bands along $k_{y}$ for $k_{x}$ $=0$ of three-layer graphene with the full interaction $(\kappa$ $=2.3$ ) for different values of $n_{t}$, with $n_{b}=-n_{t}$. The band structure for low density [Fig. 6(a)] is similar to our earlier result ${ }^{10}$ when only one gate is applied to the trilayer system. In the presence of two gates we are able to move the Fermi energy inside the opened gap. However, at intermediate and high densities the band structure is very different from our earlier result ${ }^{10}$ (see inset of Fig. 8) when only a single gate is applied.

The charge density $n_{i}$ (solid curves) on the different graphene layers is shown in Fig. 7 for the system with the full interaction with $\kappa=2.3$ and for the case when $\kappa=1$ (dashed curves) as a function of the density of the top gate $n_{t}$ (when back gate density $\left|n_{b}\right|=n_{t}$ ). Notice that for the case with $\kappa=1$ the values of the induced electron density in the outer layers are larger than for the case with $\kappa=2.3$. It is interesting that when the full interaction is included the excess density in the middle layer is no longer zero; i.e., for $\left|n_{b}\right|=n_{t}=10^{13} \mathrm{~cm}^{-2}$ and $\kappa=2.3$ the layer densities are $n_{1}=$ $-3.84, n_{2}=1.70, n_{3}=3.67$ in units of $10^{12} \mathrm{~cm}^{-2}$. This can be explained by the fact that negatively and positively charged gates (with the same magnitude of charges on them)

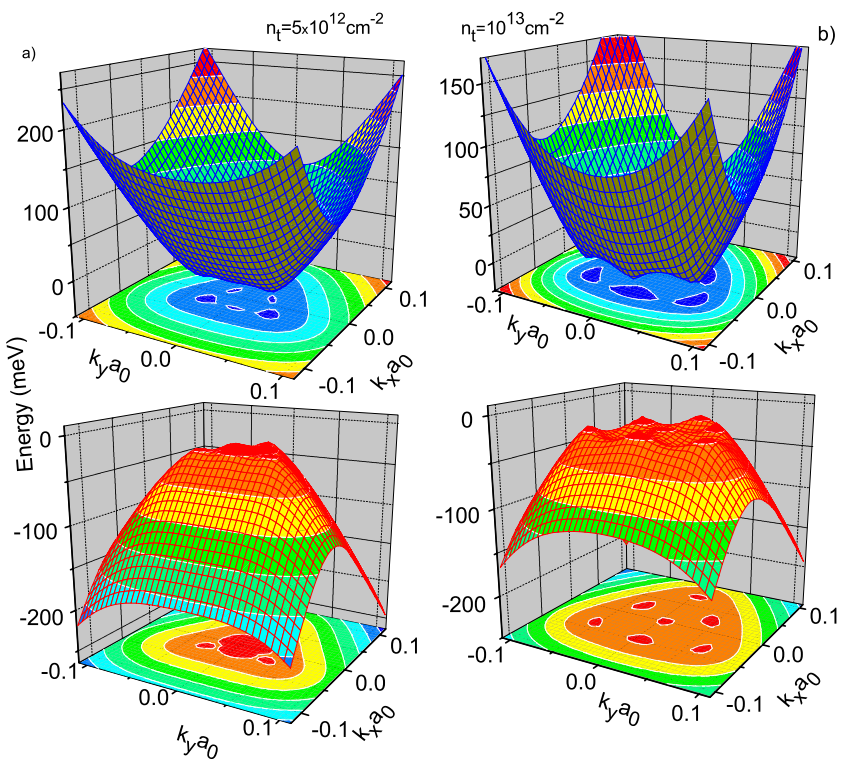

FIG. 4. (Color online) The first conduction and the highest valence band, with the corresponding contourplots for three-layer graphene near the $K$ point ( $K$ point is chosen as the origin, $\kappa=2.3$ ) with equal but opposite charges on the top and back gates: (a) when $n_{t}=\left|n_{b}\right|=5 \times 10^{12} \mathrm{~cm}^{-2}$ and (b) $n_{t}=\left|n_{b}\right|=10^{13} \mathrm{~cm}^{-2}$. The Fermi energy for both densities is located in the energy gap. 

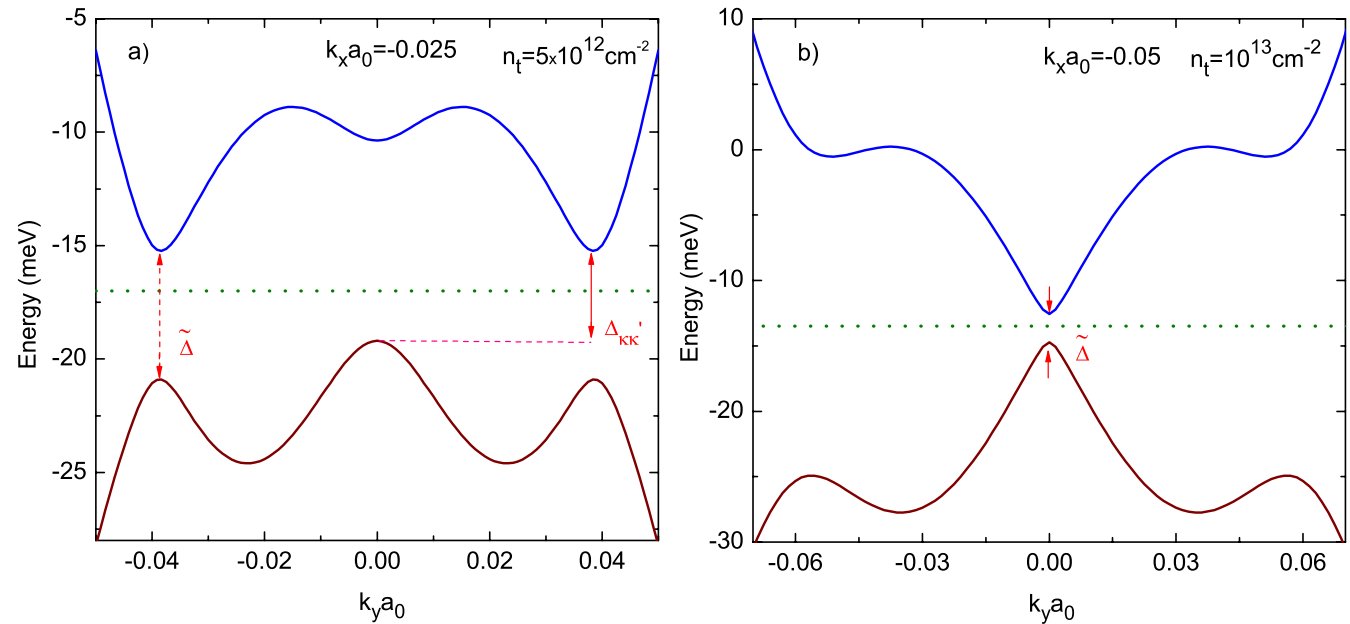

FIG. 5. (Color online) The lowest conduction and the highest valence bands of the three-layer graphene $(\kappa=2.3)$ with the full interaction as function of $k_{y}$ (around the $K$ point and with fixed $k_{x}$ ) when top and back gates are such that $n_{t}=-n_{b}$ : (a) for $k_{x} a_{0}=-0.025$ and $n_{t}=5$ $\times 10^{12} \mathrm{~cm}^{-2}$ and (b) for $k_{x} a_{0}=-0.05$ and $n_{t}=10^{13} \mathrm{~cm}^{-2}$. The Fermi energy is located in the energy gap and is indicated by the dotted curve.

induce different gaps and layer densities [see Figs. 8(a) and 9 in the next section). As a consequence of this asymmetry in the induced excess electron densities a gap opens, which is absent in the case when only $\gamma_{0}, \gamma_{1} \neq 0$. For the case when only $\gamma_{0}, \gamma_{1} \neq 0$ we found that $n_{2}=0$ and that the outer layers have equal excess densities, i.e., $n_{1}=-n_{3}$.
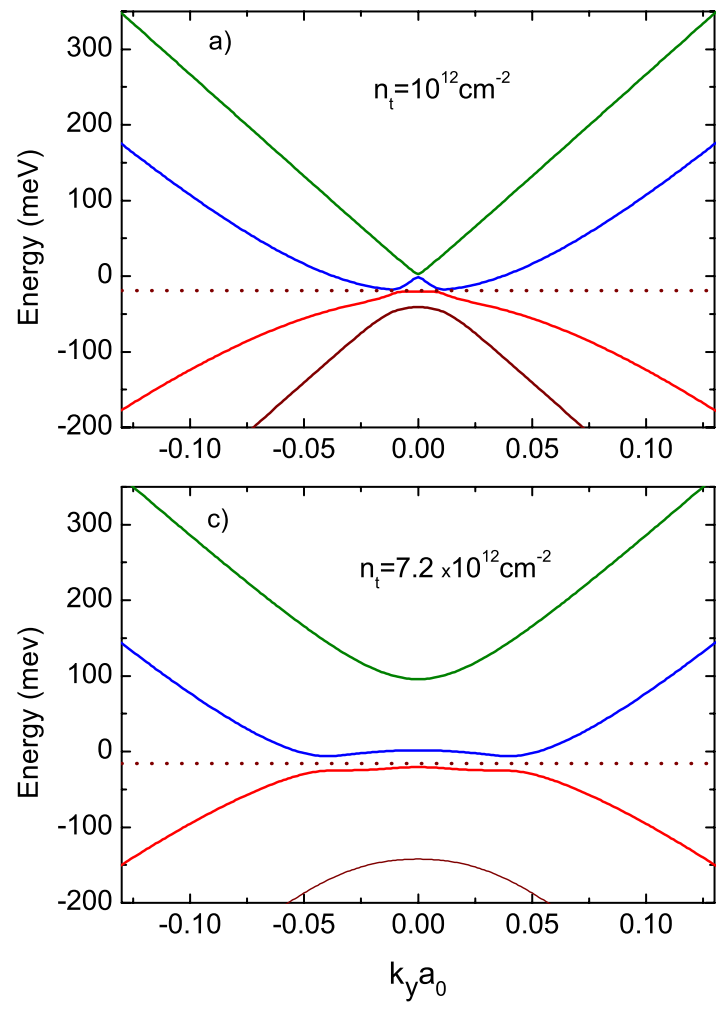

\section{Three-layer graphene in the presence of a single (top or back) gate}

Here we extend our previous work ${ }^{10}$ on the single gate tuning of the electron density in trilayer graphene to the tuning of the hole density and we will show that there is a clear asymmetry between electrons and holes. The dependence of

FIG. 6. (Color online) The band structure of three-layer graphene $(\kappa=2.3)$ with the full interaction, when top and back gates with $n_{b}$ $=-n_{t}$ are applied, as a function of $k_{y}$ (around the $K$ point, for $k_{x}=0$ ). The Fermi energy for all the densities is located in the energy gap and is indicated by the dotted curve. 


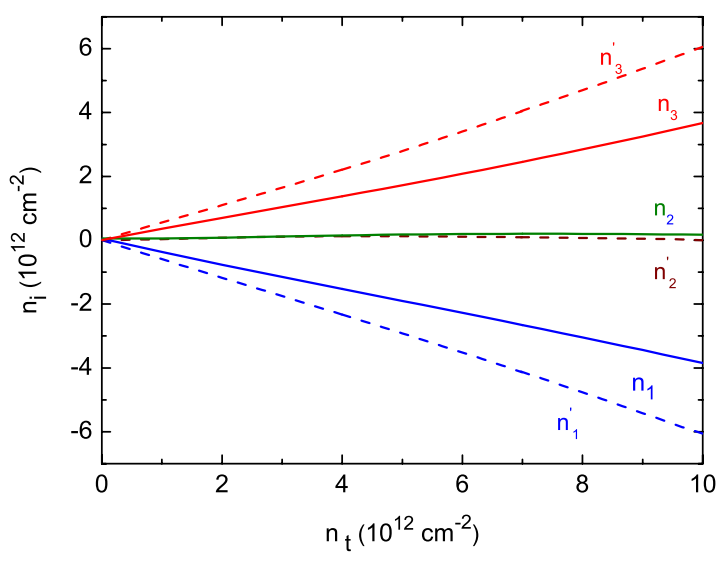

FIG. 7. (Color online) The charge density $n_{i}$ (solid curves) on the different graphene layers for the trilayer system with $\kappa=2.3$ (with the full interaction) and $n_{i}^{\prime}$ (dashed curves) for the case when $\kappa=1$ as a function of the charge density on the top gate $n_{t}$ (the back gate density $n_{b}=-n_{t}$ ).

the energy gap at the $K$ point $\Delta_{0}$ and of the true direct gap $\widetilde{\Delta}$ (solid curve) as well as the true indirect gap $\Delta_{k k^{\prime}}$ (dotted curve, which for $n>1.8 \times 10^{12} \mathrm{~cm}^{-2}$ coincides with $\widetilde{\Delta}$ ) are shown in Fig. 8(a), as a function of the total density of excess electrons $n=n_{1}+n_{2}+n_{3}$ when only the top or back gate is applied for $\kappa=1$. In the region when excess densities are negative (in the presence of the top gate) the Fermi energy is located in the valence band. Notice that the energy gaps are: (i) not symmetric around $n=0$, (ii) they have a nonmonotonic behavior as a function of $n$, and (iii) for a negatively charged gate the energy bands have a "Mexican-hat" shape and due to this the energy gap at the $K$ point does not coincide with the true gap in this region of total density. Notice that the gaps for negative and positive densities are symmetric around $n=0$ for the case when only $\gamma_{0}, \gamma_{1} \neq 0$. In the case, when we take into account all the interactions between the

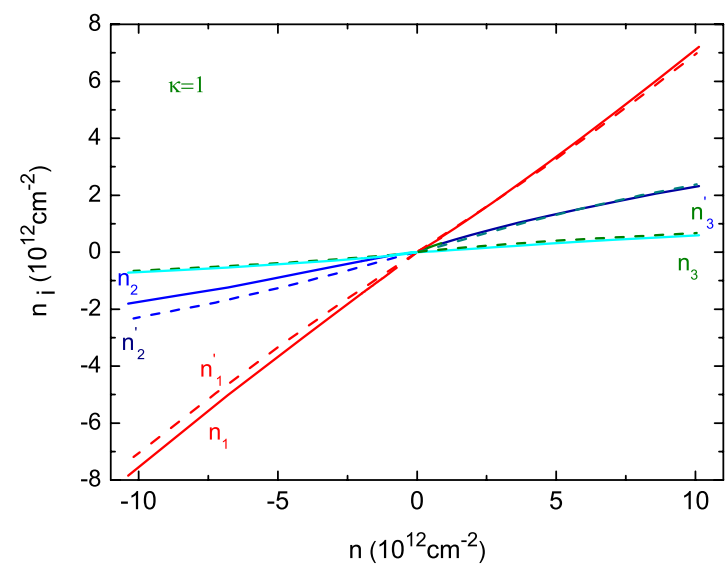

FIG. 9. (Color online) The layer densities $n_{i}$ (solid curves) for the three-layer system with the full interaction $(\kappa=1)$ and $n_{i}^{\prime}$ (dashed curves) for the case when only $\gamma_{0}, \gamma_{1}$ are different from zero, as a function of total excess density $n$ when either the back gate either the top one is applied.

different atoms except $\gamma_{3}$ the energy bands are circular symmetric. The dependence of the true gap (dashed curve) for this case is shown in Fig. 8(b) when $\kappa=2.3$. Notice that the true gap remains zero up to the density $n \approx 2 \times 10^{12} \mathrm{~cm}^{-2}$. The parameter $\gamma_{3}$ describes the interaction of $B$-type atoms between adjacent layers (which are not on top of each other) which is not well known for multilayers of graphene and we present in Fig. 8(b) the results for $\widetilde{\Delta}$ for different values of $\gamma_{3}$ : (i) $\gamma_{3}=0.15$ (dot-dashed curve) and (ii) $\tilde{\Delta}$ when $\gamma_{3}$ $=0.29$ (solid curve). It is clear that the gap is strongly influenced by the value of $\gamma_{3}$. The indirect true gap $\Delta_{k k^{\prime}}$ is also presented (dotted curves) in Fig. 8(b) for $\gamma_{3}=0.29$ and is very small for $n<5.5 \times 10^{12} \mathrm{~cm}^{-2}$. For larger densities this gap increases almost linearly with the density. The inset to this figure shows the band structure of the three-layer system calculated with the full interaction along $k_{y}\left(k_{x}=0\right)$ for the
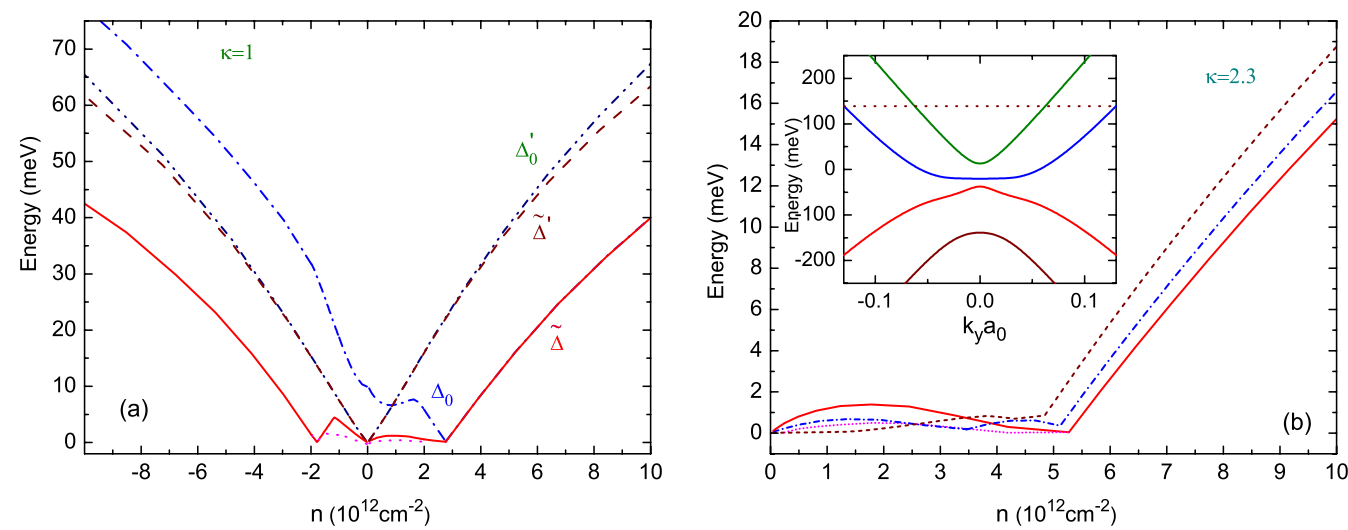

FIG. 8. (Color online) Density dependence of the energy gaps in trilayer graphene when only one gate is applied: (a) $\Delta_{0}$ at the $K$ point (dot-dashed curve), the true direct gap $\widetilde{\Delta}$ (solid curve), and the true indirect gap (dotted curve) $\Delta_{k k^{\prime}}$ for $\kappa=1$ when including the full interaction. For comparison we show also the corresponding results, $\Delta_{0}^{\prime}$ (dot-dot-dashed curve) and $\widetilde{\Delta^{\prime}}$ (dashed curve) when only $\gamma_{0}, \gamma_{1}$ $\neq 0$. (b) The dependence of the true gap (dashed curve) for the case when only $\gamma_{3}=0$ and when all the interactions are included with $\gamma_{3}$ $=0.15$ (dot-dashed curve) for $\kappa=2.3$. We show also the corresponding results for $\gamma_{3}=0.29$ for the true direct gap (solid curve) and the true indirect gap $\Delta_{k k^{\prime}}$ (dotted curve). Inset: the band structure of the three-layer system calculated with the full interaction (for $\left.\gamma_{3}=0.29\right)$ along $k_{y}$ for $k_{x}=0$ with $n=2 \times 10^{12} \mathrm{~cm}^{-2}$; the dotted curve is the Fermi level. 

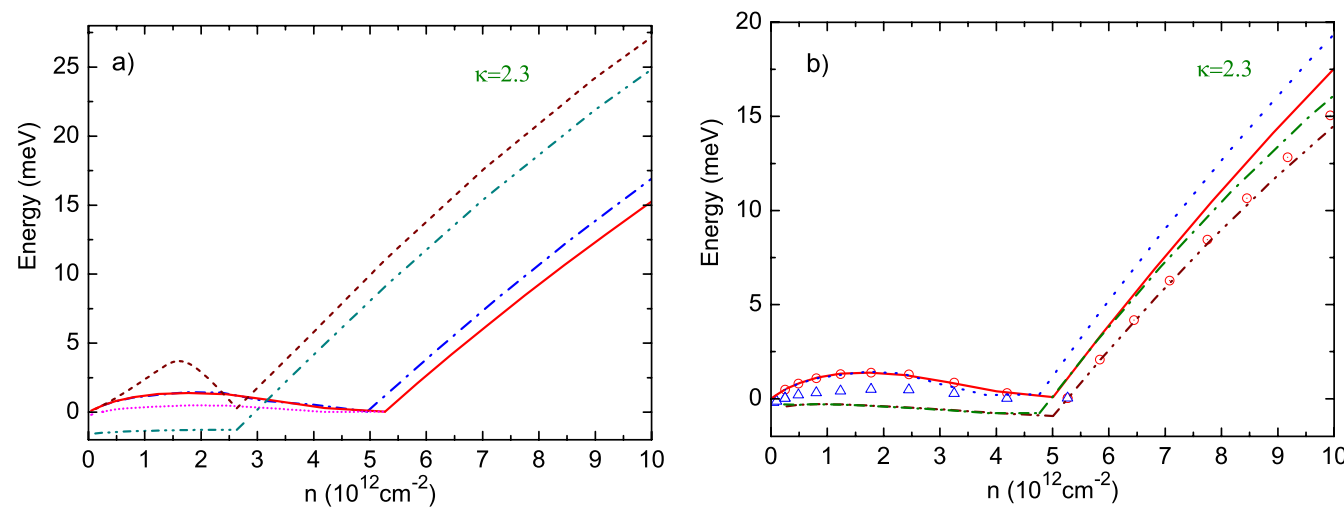

FIG. 10. (Color online) The dependence of the true direct and indirect gaps as a function of the total excess electron density $n$ for three-layer graphene with $\kappa=2.3$ : (a) $\widetilde{\Delta}$ (solid curve) and $\Delta_{k k^{\prime}}$ (dotted curve) obtained with SWMcC parameters; $\widetilde{\Delta}$ (dot dashed) for the case when signs of $\gamma_{0}, \gamma_{1}$ are changed; $\widetilde{\Delta}$ (dashed curve); and $\Delta_{k k^{\prime}}$ (dot-dot-dashed curve) when $\gamma_{2}$ sign is changed. (b) $\widetilde{\Delta}$ (solid curve) and $\Delta_{k k^{\prime}}$ (dot-dot-dashed curve) for the case $\gamma_{0}$ sign is changed; $\widetilde{\Delta}$ (dashed curve) and $\Delta_{k k^{\prime}}$ (dot-dashed curve) if the sign of $\gamma_{1}$ is changed. For comparison purposes $\widetilde{\Delta}$ for the SWMcC parameters is indicated by circles and $\Delta_{k k^{\prime}}$ is given by triangulares.

total electron density $n=8 \times 10^{12} \mathrm{~cm}^{-2}$, induced by a positively charged back gate. Comparing Figs. 8(a) and 8(b) we notice that the gaps for the system with $\kappa=2.3$ at high densities are approximately half the gaps obtained for $\kappa=1$.

The distribution of the charge density over the different layers is shown in Fig. 9 for the system with $\kappa=1$ and when the full interaction is included (solid curves), as well as for the case when only $\gamma_{0}, \gamma_{1} \neq 0$ (dashed curves). Notice that in the negative gate voltage region (when a top gate is present and the total excess electronic densities are negative) the inclusion of the full interaction affects much stronger the density redistribution of the individual layers than in the case when only the back gate is applied to the system.

Since the SWMcC parameters are not known for multilayers of graphene and the maximal change in these parameters takes place when changing their signs, in the rest part of this section we will study the dependence of the signs of the different SWMcC parameters on the band structure of the trilayer. We found that when we change the signs of $\gamma_{0}, \gamma_{1}, \gamma_{3}, \gamma_{4}$ the true and $K$-point gaps shift but conserve the oscillatorylike behavior of the gaps as a function of $n$. The change in the sign of $\gamma_{2}$ modifies the band structure more strongly. We found the following interesting fact: when one changes simultaneously the sings for some SWMcC parameters, then the true and $K$-point gaps remain unaltered. For example, the true gap obtained for the case when the signs of $\gamma_{0}$ and $\gamma_{4}$ (or $\gamma_{0}, \gamma_{1}, \gamma_{3}$ ) are simultaneously changed coincides with that we found using the SWMcC parameters (see Ref. 14) for graphite, i.e., the results corresponding to the solid curve in Fig. 8(b). The true gap $\widetilde{\Delta}$ (solid curve) obtained for SWMcC parameters and the true indirect gap $\Delta_{k k^{\prime}}$ (dotted curve) are also presented in Fig. 10(a) for $\kappa=2.3$. Change in $\gamma_{0}, \gamma_{3}, \gamma_{4}$ gives the same result as one changes $\gamma_{0}, \gamma_{1} ; \widetilde{\Delta}$ for this case (dot-dashed curve) is presented in Fig. 10(a). The true direct- and indirect-gap behaviors for this case are close to the corresponding gaps found with SWMcC parameters until $n \approx 5 \times 10^{12} \mathrm{~cm}^{-2}$. The change in the sign of $\gamma_{3}$ leads to similar dependencies as if the signs of $\gamma_{0}, \gamma_{1}$ are changed and for large densities in both cases the indirect gap coincides with the direct one.
A change in sign of $\gamma_{2}$ and a simultaneous change in sign of $\gamma_{0}, \gamma_{1}, \gamma_{4}$ (or $\gamma_{0}, \gamma_{3}$ ) leads to the same gaps as if only $\gamma_{2}$ is changed; the true gap $\widetilde{\Delta}$ (dashed curve) and $\Delta_{k k^{\prime}}$ (dot-dotdashed curve) are presented in Fig. 10(a). One can see that in the case when the sign of $\gamma_{2}$ is changed then the indirect gap is negative until $n \approx 3 \times 10^{12} \mathrm{~cm}^{-2}$ due to the fact that the conduction and valence bands overlap for certain momenta. We see also that in this case the indirect gap does not coincide with the true gap at high densities.

Change in the signs of $\gamma_{0}, \gamma_{1}, \gamma_{3}, \gamma_{4}$ gives the same result as one changes $\gamma_{0}$ and $\widetilde{\Delta}$ (solid curve) as well as $\Delta_{k k^{\prime}}$ (dotdot-dashed curve) for this case are shown in Fig. 10(b). As a comparison $\widetilde{\Delta}$ for the SWMcC parameters is indicated by circles in Fig. 10(b) and $\Delta_{k k^{\prime}}$ is given by triangulares. Also, a change in signs of $\gamma_{0}, \gamma_{1}, \gamma_{4}$ or $\gamma_{0}, \gamma_{3}$ leads to the same result as if the sign of $\gamma_{1}$ is changed and for this case $\tilde{\Delta}$ (dashed curve) as well as $\Delta_{k k^{\prime}}$ (dot-dashed curve) are given in Fig. 10(b). Notice that: (i) in both cases when $\gamma_{0}$ or $\gamma_{1}$ are changed, then the true direct and indirect gaps do not coincide at high densities and (ii) there is a shift between the true

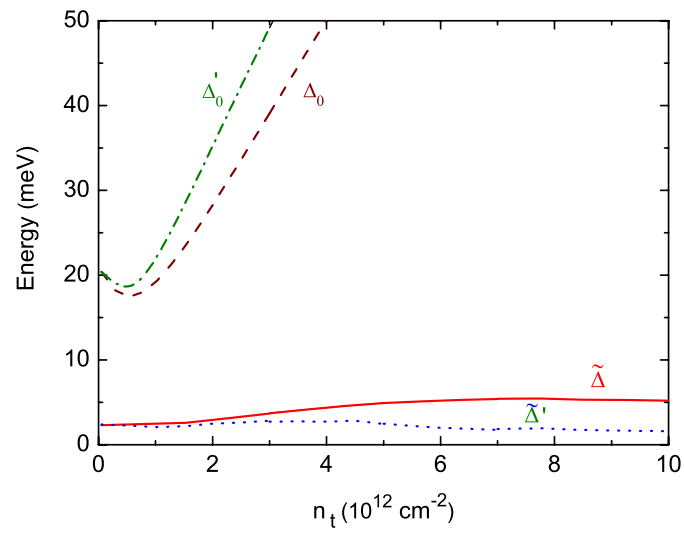

FIG. 11. (Color online) The gap $\Delta_{0}$ at the $K$ point (dashed curve) and the true gap $\widetilde{\Delta}$ (solid curve) as a function of the top gate $n_{t}=-n_{b}$ for four-layer graphene with $\kappa=2.3$, and for $\kappa=1$ where $\Delta_{0}^{\prime}$ (dot-dashed curve) and $\widetilde{\Delta}^{\prime}$ (dotted curve). 

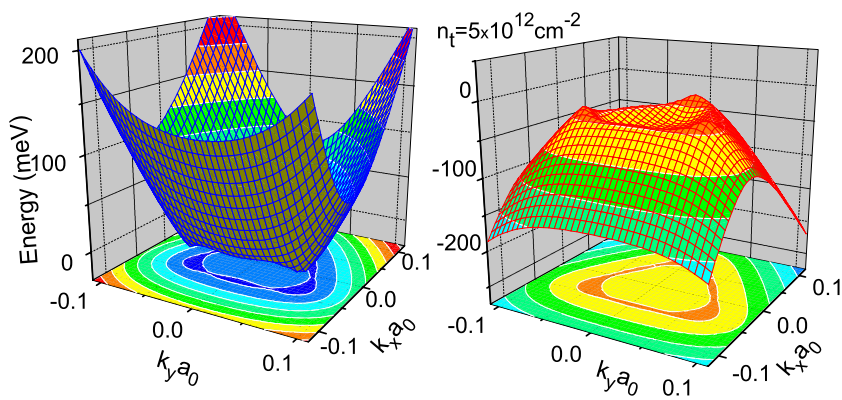

FIG. 12. (Color online) The first conduction (left figure) and the highest valence (right figure) bands, and the corresponding contourplots for the four-layer graphene near the $K$ point ( $K$ point is chosen as the origin, $\kappa=2.3)$ when $n_{t}=-n_{b}=5 \times 10^{12} \mathrm{~cm}^{-2}$.

gaps for the cases when $\gamma_{0}$ or $\gamma_{1}$ are changed. Therefore, in an experiment one should be able to observe a nonmonotonic behavior of the energy gaps, from which one can deduce the true value for the SWMcC parameters.

\section{FOUR-LAYER GRAPHENE SYSTEM IN AN EXTERNAL ELECTRIC FIELD}

In this section we consider a four-layer graphene system with top and back gates, which induce a total excess density $n=n_{1}+n_{2}+n_{3}+n_{4}$, where $n_{i}$ is the excess density on the $i$ th layer as counted from the top gate. The corresponding change in the potential energy between consecutive layers is

$$
\Delta_{1,2}(n)=\alpha\left(n_{2}+n_{3}+n_{4}-\left|n_{b}\right|\right),
$$

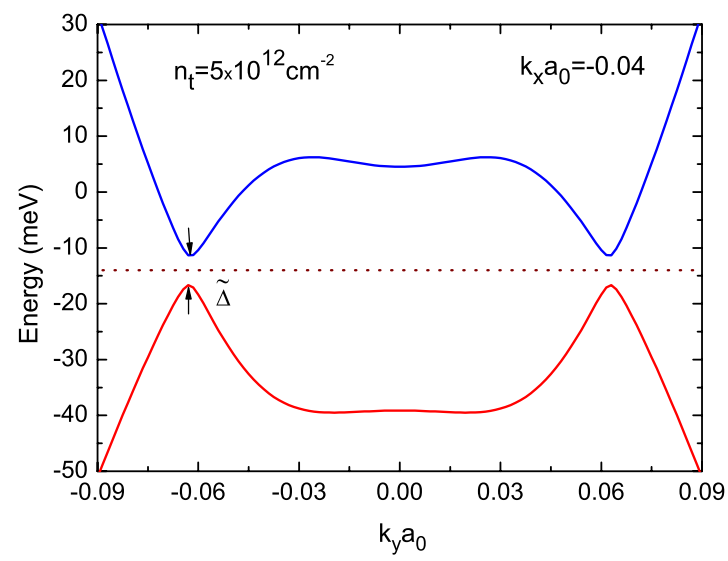

FIG. 13. (Color online) The lowest conduction and the highest valence bands of the four-layer graphene $(\kappa=2.3)$ with the full interaction as function of $k_{y}$ (around the $K$ point) when $n_{t}=-n_{b}=5$ $\times 10^{12} \mathrm{~cm}^{-2}$ and $k_{x} a_{0}=-0.04$. The Fermi energy (dotted curve) is located in the energy gap.

$$
\begin{gathered}
\Delta_{2,3}(n)=\alpha\left(n_{3}+n_{4}-\left|n_{b}\right|\right), \\
\Delta_{3,4}(n)=\alpha\left(n_{4}-\left|n_{b}\right|\right) .
\end{gathered}
$$

By adding $\Delta^{I I}=\Delta_{1,2}(n), \quad \Delta^{I I I}=\Delta_{1,2}(n)+\Delta_{2,3}(n), \quad$ and $\Delta^{I V}$ $=\Delta_{1,2}(n)+\Delta_{2,3}(n)+\Delta_{3,4}(n)$ to the on-site elements of the II, III, and IV layers, respectively, of the Hamiltonian in the absence of the gates, we obtain the Hamiltonian for the four $A B$-stacked graphene layers in the presence of top and bottom gates

$$
\left(\begin{array}{cccccccc}
\Delta^{\prime} & \gamma_{0} f & \gamma_{1} & -\gamma_{4} f^{*} & \gamma_{5} / 2 & 0 & 0 & 0 \\
\gamma_{0} f^{*} & \gamma_{2} & -\gamma_{4} f^{*} & \gamma_{3} f & 0 & \gamma_{2} / 2 & 0 & 0 \\
\gamma_{1} & -\gamma_{4} f & \Delta^{I I}+\Delta^{\prime} & \gamma_{0} f^{*} & \gamma_{1} & -\gamma_{4} f & \gamma_{5} / 2 & 0 \\
-\gamma_{4} f & \gamma_{3} f^{*} & \gamma_{0} f & \Delta^{I I}+\gamma_{2} & -\gamma_{4} f & \gamma_{3} f^{*} & 0 & \gamma_{2} / 2 \\
\gamma_{5} / 2 & 0 & \gamma_{1} & -\gamma_{4} f^{*} & \Delta^{I I}+\Delta^{\prime} & \gamma_{0} f & \gamma_{1} & -\gamma_{4} f^{*} \\
0 & \gamma_{2} / 2 & -\gamma_{4} f^{*} & \gamma_{3} f & \gamma_{0} f^{*} & \Delta^{I I I}+\gamma_{2} & -\gamma_{4} f^{*} & \gamma_{3} f \\
0 & 0 & \gamma_{5} / 2 & 0 & \gamma_{1} & -\gamma_{4} f & \Delta^{I V}+\Delta^{\prime} & \gamma_{0} f^{*} \\
0 & 0 & 0 & \gamma_{2} / 2 & -\gamma_{4} f & \gamma_{3} f^{*} & \gamma_{0} f & \Delta^{I V}+\gamma_{2}
\end{array}\right) \text {, }
$$

where $\Delta^{\prime}=\Delta+\gamma_{5}$. The eight coefficients $c_{A_{i}}=c_{A_{i}}(\vec{k})$ and $c_{B_{i}}$ $=c_{B_{i}}(\vec{k})$, for fixed values of the layer asymmetries defined by Eqs. (8)-(10), can be obtained by diagonalizing Eq. (11). The electronic densities on the individual layers are given by Eq. (6). The gaps $\Delta_{0}$ and $\widetilde{\Delta}$ are evaluated self-consistently analogously as was done for the three-layer system.

The variation in $\widetilde{\Delta}$ (solid curve) and $\Delta_{0}$ (dashed curve) with $n_{t}$ (charges on the top and back gates are taken opposite but equal in magnitude) are shown in Fig. 11 for $\kappa=2.3$ and when the full interaction between the atoms is included. The same gaps $\Delta_{0}^{\prime}$ (dot-dashed curve) and $\widetilde{\Delta^{\prime}}$ (dotted curve) are shown for the case $\kappa=1$.

Figure 12 shows typical 3D plots and corresponding contourplots of the first conduction and the highest valence band for the four-layer graphene $(\kappa=2.3)$ for an intermediate value of $n_{t}=-n_{b}=5 \times 10^{12} \mathrm{~cm}^{-2}$. Notice that the valence band has a local broad minimum at the $K$ point which is surrounded by three local maxima. Figure 13 gives the band structure along $k_{y}$ for $k_{x}=-0.04$. In this plane the minimal gap equals the true direct gap. The maxima in the highest valence band is located just below the lowest conduction-band minima (see 


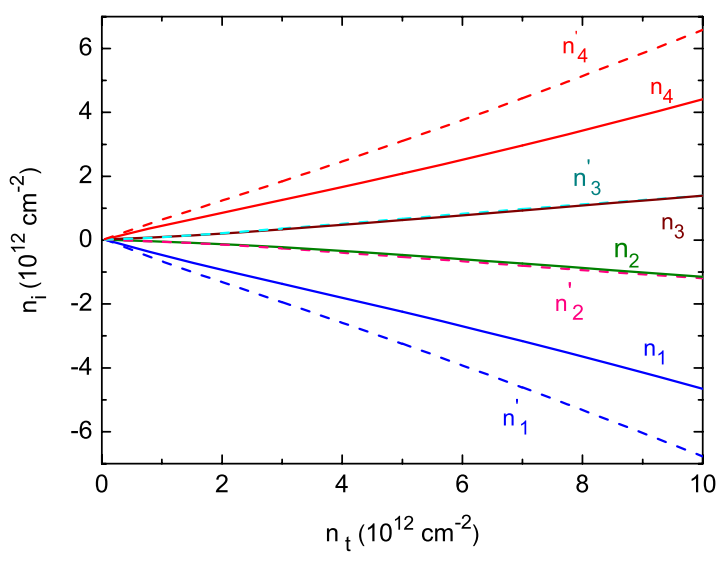

FIG. 14. (Color online) The layer densities $n_{i}$ (solid curves) for the four-layer system with $\kappa=2.3$ and $n_{i}^{\prime}$ (dashed curves) for the case when $\kappa=1$ (in both cases the full interaction is included) as a function of the charge density on the top gate $n_{t}=-n_{b}$.

Fig. 13) and due to this the indirect gap coincides with the direct one for the four-layer system. Notice that the true gap is found for $k_{y}, k_{x} \neq 0$ and is much smaller than the gap near the $K$ point.

Figure 14 shows the distribution of the charge density over the different graphene layers $n_{i}$ (solid curves) when $\kappa$ $=2.3$ and $n_{i}^{\prime}$ (dashed curves) for the case when $\kappa=1$ as a function of the top gate density $n_{t}=-n_{b}$ (the full interaction is included in both cases). We found $n_{i} \approx n_{i}^{\prime}$ for $i=2,3$ although the potential-energy difference between these layers for the case $\kappa=2.3$ is twice smaller than for $\kappa=1$. This is even more remarkable because the true gap $\widetilde{\Delta}=5.1 \mathrm{meV}$ for $\left|n_{b}\right|=n_{t}$ $=10^{13} \mathrm{~cm}^{-2}$ is more than three times larger when $\kappa=2.3$ than the corresponding gap $\widetilde{\Delta^{\prime}}=1.6 \mathrm{meV}$ for $\kappa=1$. In order to understand this we consider also the case when only $\gamma_{0}, \gamma_{1}$ $\neq 0$, where the true gap is almost three times larger than the corresponding gap for the full interaction case. We found that $\widetilde{\Delta}=15.6 \mathrm{meV}$ for $n_{t}=-n_{b}=10$ (in units of $10^{12} \mathrm{~cm}^{-2}, \kappa$ $=2.3$ ) and corresponding induced densities on the outer (on the inner) layers are symmetric $\left|n_{1}\right|=n_{4}=4.40 \quad\left(\left|n_{2}\right|=n_{3}\right.$ $=1.41)$ while for the same fixed parameters in the case of the full interaction $n_{1}=-4.66, n_{2}=-1.15, n_{3}=1.39, n_{4}=4.41$. Also, in the case when only $\gamma_{0}, \gamma_{1} \neq 0$ the increase in $\kappa$ leads to an increase in the true gap. We see that in the four-layer case the asymmetry in the induced density between the inner layers makes the gap smaller. We conclude that the increase in $\kappa$, which results in an increase in screening, effectively suppresses the layer asymmetry as well as the density asymmetry between the second and third layers. This is responsible for the increase in the gap with increase in $\kappa$.

In Ref. 10 it was found that when only one gate was present the true gap had the tendency to decrease with increasing number of graphene layers. This behavior is connected with the excess charge distribution between the layers and corresponding asymmetries between the graphene layers. Due to imperfect screening, the charge density on the layers furthest from the gate is considerably smaller in comparison with the excess density on the layer closest to the gate as shown in Fig. 9. With the increase in the number of layers, the asymmetry between the last layers abruptly decreases,

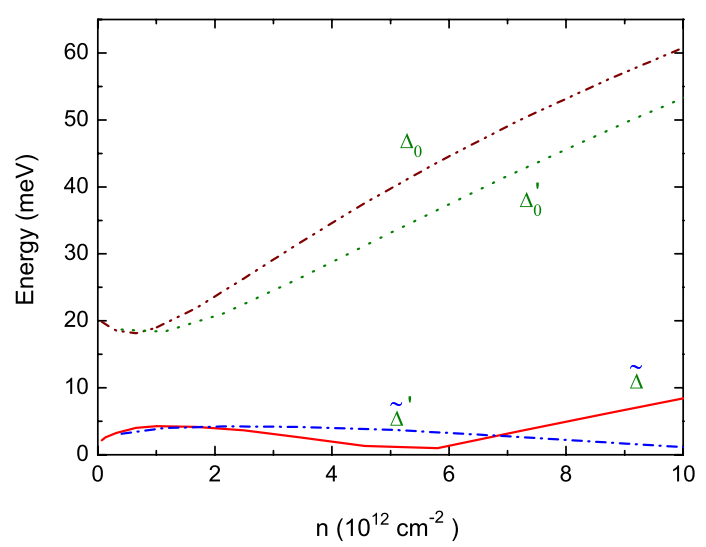

FIG. 15. (Color online) The gap $\Delta_{0}$ at the $K$ point (dot-dotdashed curve) and the true gap $\tilde{\Delta}$ (solid curve) as a function of the total excess electron density $n$ when only the back gate is applied for the value of $\kappa=1$ for a four-layer graphene when including the full interaction. The corresponding results for $\kappa=2.3$ are shown for $\Delta_{0}^{\prime}$ (dotted curve) and $\widetilde{\Delta^{\prime}}$ (dash-dotted curve).

resulting in layers with almost no excess charge. Also, when only one gate is applied to the system we found that with increasing $\kappa$ the true gap decreases as shown in Fig. 15.

Now, when top and back gates are applied with opposite (but equal in magnitude) charges on them, in order to open an energy gap and tune the Fermi energy into the energy gap, we found that for $\kappa=2.3$ the gap for four layers of graphene at high densities is larger than the gap for the three-layer system. This fact is a consequence of the presence of Dirac fermions in $A B$-stacked graphene multilayers with an odd number of layers and that for an even number of stacked graphene layers only charge carriers with a parabolic energy dispersion exist. ${ }^{12}$

\section{CONCLUSIONS}

We considered multilayers of graphene in the field of top and back gates, which are negatively and positively charged, respectively, and investigated the electronic structure near the $K$ point. For three layers of graphene we found a true indirect gap, which at high densities coincides with the direct true gap. For the three-layer system with inclusion of full interaction the true energy gap $\widetilde{\Delta}$ as well as the true indirect gap $\Delta_{k k^{\prime}}$ have a nonmonotonous behavior as a function of the potential on the top gate, provided that charges on the top and back gates are opposite but equal in magnitude. The Fermi energy, in this case, is always located in the energy gap. It is interesting that, when both gates are applied to the three-layer system, increasing $\kappa$ leads to a reduction in the gap at high densities and for certain values of the gate voltage the gap can be closed.

We found that when only one gate is applied to a multilayer of graphene, the true gap decreases with increasing $\kappa$. However, the true gap is larger when top and back gates are applied to four layers of graphene with $\kappa=2.3$ than for the case of $\kappa=1$.

For the four-layer graphene system the gap for $\kappa=2.3$ including the full interaction is larger at high densities than 
the corresponding gap for the three-layer system when the magnitude of the charge on the top and back gates is the same. For this case, the gaps are a monotonic function of the density for even number of layers. The true gap for bilayer graphene is much larger than for the three- and the four-layer systems.

The effect of the circular asymmetry that arisen as a consequence of the trigonal warping, strongly modifies the behavior of the induced electronic gap even when the total density of induced electrons on the layers is low.

In a recent experimental work by Craciun et al. ${ }^{16}$ the resistance of trilayer graphene was measured as a function of the voltage on the top and bottom gates. They found that the resistance was maximal when $V_{b} \simeq-\alpha V_{t}$, where $V_{b}\left(V_{t}\right)$ is the back (top) gate voltage and $\alpha$ a leverage factor taking into account the difference in distance between the back and the top gates to the graphene trilayer. This result implies that the resistance is maximal when $n_{t}=-n_{b}$ in which case we find the opening of a gap with the Fermi energy inside the gap. They could understand these experimental results by assuming that trilayer graphene is a semimetal where the overlap between the electron and hole bands could be tuned by the gate voltage $V_{b}+\alpha V_{t}$. Our theoretical results provide an alternative explanation to these experimental observations. From measurements on single layer graphene ${ }^{17}$ we know that disorder is large and that around the Dirac point there are electron-density fluctuations (i.e., puddles) of size of about $10^{11} \mathrm{~cm}^{-2}$ which corresponds to very large potential fluctuations, i.e., tens of meV. From the mobility measurements on trilayer graphene ${ }^{16}$ we expect a similar type of disorder which is much larger than the here predicted gap of a few meV. Therefore, unlike bilayer graphene, for trilayer graphene gap opening is too small to be seen in the transport experiment of Ref. 16. Disorder can be reduced by using suspended graphene which is a possible rout to observe the here predicted energy gap in trilayer graphene.

\section{ACKNOWLEDGMENTS}

This work was supported by the Flemish Science Foundation (FWO-Vl), the "Belgian Science Policy" IAP program, and the Brazilian Science Foundation CNPq. One of us (A. A. A.) is supported from the Belgian Federal Science Policy Office.

\footnotetext{
*artak.avetisyan@ua.ac.be

†bart.partoens@ua.ac.be

*francois.peeters@ua.ac.be

${ }^{1}$ K. S. Novoselov, A. K. Geim, S. V. Morozov, D. Jiang, M. I. Katsnelson, I. V. Grigorieva, S. V. Dubonos, and A. A. Firsov, Nature (London) 438, 197 (2005).

${ }^{2}$ A. K. Geim and K. S. Novoselov, Nature Mater. 6, 183 (2007).

${ }^{3}$ P. Blake, P. D. Brimicombe, R. R. Nair, T. J. Booth, D. Jiang, F. Schedin, L. A. Ponomarenko, S. V. Morozov, H. F. Gleeson, E. W. Hill, A. K. Geim, and K. S. Novoselov, Nano Lett. 8, 1704 (2008).

${ }^{4}$ Y. Zhang, Y.-W. Tan, H. L. Stormer, and P. Kim, Nature (London) 438, 201 (2005).

${ }^{5}$ C. Berger, Z. Song, X. Li, X. Wu, N. Brown, C. Naud, D. Mayou, T. Li, J. Hass, A. N. Marchenkov, E. H. Conrad, Ph. N. First, and W. A. de Heer, Science 312, 1191 (2006).

${ }^{6}$ I. A. Luk'yanchuk and Y. Kopelevich, Phys. Rev. Lett. 93, 166402 (2004); 97, 256801 (2006).

${ }^{7}$ E. McCann, Phys. Rev. B 74, 161403(R) (2006).
}

${ }^{8}$ E. V. Castro, K. S. Novoselov, S. V. Morozov, N. M. R. Peres, J. M. B. Lopes dos Santos, J. Nilsson, F. Guinea, A. K. Geim, and A. H. Castro Neto, Phys. Rev. Lett. 99, 216802 (2007).

${ }^{9}$ J. B. Oostinga, H. B. Heersche, X. Liu, A. F. Morpurgo, and L. M. K. Vandersypen, Nature Mater. 7, 151 (2008).

${ }^{10}$ A. A. Avetisyan, B. Partoens, and F. M. Peeters, Phys. Rev. B 79, 035421 (2009).

${ }^{11}$ M. Koshino and E. McCann, Phys. Rev. B 79, 125443 (2009).

${ }^{12}$ B. Partoens and F. M. Peeters, Phys. Rev. B 75, 193402 (2007).

${ }^{13}$ J. Guttinger, C. Stampfer, F. Molitor, D. Graf, T. Ihn, and K. Ensslin, New J. Phys. 10, 125029 (2008).

${ }^{14}$ B. Partoens and F. M. Peeters, Phys. Rev. B 74, 075404 (2006).

${ }^{15}$ L. M. Malard, J. Nilsson, D. C. Elias, J. C. Brant, F. Plentz, E. S. Alves, A. H. Castro Neto, and M. A. Pimenta, Phys. Rev. B 76, 201401(R) (2007).

${ }^{16}$ M. F. Craciun, S. Russo, M. Yamamoto, J. B. Oostinga, A. F. Morpurgo, and S. Tarucha, Nat. Nanotechnol. 4, 383 (2009).

${ }^{17}$ J. Martin, N. Akerman, G. Ulbricht, T. Lohmann, J. H. Smet, K. von Klitzing, and A. Yacoby, Nat. Phys. 4, 144 (2008). 